\title{
Determinantes que inciden en la calidad de rendimiento académico de los estudiantes de ingeniería
}

\author{
Sergio Junior Navarro Hudiel ${ }^{1}$ \\ Sandra Lorena Blandón Navarro²
}

\section{RESUMEN}

Los factores determinantes del rendimiento académico para las instituciones educativas representan un proceso complejo. Muchos de los aspectos abordados en el presente ensayo hacen referencia a la interacción multidimensional entre elementos de carácter personal, institucional, socio económico así como tecnológico. Esta revisión destaca la importancia del análisis y evaluación del rendimiento académico para los formados en la ingeniería y la necesidad de evaluar los niveles de empleabilidad que permitan valorar el aporte de las instituciones de educación superior en su entorno, pudiendo así realizar políticas tanto a corto, mediano y largo plazo que permitan aumentar las posibilidades para el empleo de sus estudiantes. Las acciones estratégicas propuestas deberán de incluir el vínculo de las universidades con los distintos sectores que dinamizan las economías de modo que en conjunto pueden resolverse problemáticas a nivel empresarial, local y nacional que se traduzca en elementos importantes del desarrollo local.

Palabras clave: rendimiento académico, factores determinantes, empleabilidad, Ingeniería.

Recibido: 19 de septiembre de 2017

Aceptado: 04 de diciembre de 2017

1 Profesor Titular, Universidad Nacional de Ingeniería, Sede Regional Norte, Estelí, estudiante del Doctorado en Gestión y Calidad de la Investigación Científica; UNAN Managua; Correo electrónico: sergio.navarro@norte.uni. edu.ni

2 Profesora Titular, Universidad Nacional de Ingeniería, Sede Regional Norte, Estelí, Doctora en Ciencias de la Ingeniería de Alimentos (Universidad de Sao Paulo), Correo electrónico: sandra.blandon@norte.uni.edu.ni 


\title{
Factors that affect the quality of academic performance of engineering students
}

\begin{abstract}
The factors that determine the academic performance in educational institutions represent a complex process. Many of the aspects addressed in this essay refer to the multidimensional interaction between personal, institutional, socio-economic as well as technological elements. It highlights the importance of the analysis and evaluation of academic performance for those trained in engineering and the need to evaluate the levels of employability that allow to assess the contribution of higher education institutions in their environment, Medium and long term that increase the possibilities for the employment of its students. The proposed strategic actions should include the link between the universities and the different sectors that boost economy so that jointly they can solve problems at the enterprise level, local and national, therefore, become into important elements of local development.
\end{abstract}

Keywords: academic performance, factors, employability, Engineering.

\section{INTRODUCCIÓN}

La Ingeniería es una profesión orientada hacia la aplicación competente de un cuerpo distintivo de conocimientos, basado en la matemática, las ciencias naturales y la tecnología, integradas con la gestión empresarial, que se adquiere mediante la educación y la formación profesional. La Ingeniería está orientada hacia el desarrollo, provisión y manutención de infraestructura, bienes y servicios para la industria y la comunidad (CINDA, 2004, pág. 179).

Es necesario realizar caracterización de los factores que contribuyen en el rendimiento académico de los estudiantes de ingeniería y su relación con índices de empleabilidad, tomando en cuenta el entorno local y los factores, causas y posibles acciones para mejorar el desempeño académico y los niveles de empleabilidad de los egresados, basado en experiencias exitosas desarrolladas a nivel internacional, nacional y local. Los resultados de esta revisión podrán servir como referencia en la generación de estrategias y políticas para la mejora del rendimiento académico de los estudiantes de ingeniería. También puede ser de utilidad en la elaboración de un plan de acción que contribuya a corto, mediano y largo plazo, a mejorar el rendimiento académico y los niveles de empleabilidad de los estudiantes de las diferentes carreras de profesionalización. Se espera que las vivencias y experiencias de otros entornos puedan ser adecuadas para investigaciones educativas de las universidades en correspondencia con la realidad actual. 


\section{DESARROLLO}

En referencia a lo indicado por Pedroza (2016), Irigoin y Fernando ( 2002) y otros, el mundo moderno es cada vez más complejo, marcado por los cambios tecnológicos y en las conductas humanas, indicando mayores exigencias de la productividad y de la calidad. Las universidades no están ajenas a estos cambios, donde la sociedad demanda calidad en la educación para formar profesionales integrales con valores, habilidades y actitudes para el mercado laboral cada vez más competitivo y selectivo. En ese sentido, la sociedad espera que los graduados universitarios posean capacidad de interrelacionarse, de trabajar en equipo y de adaptarse a los cambios. Las instituciones educativas están llamadas a contribuir en la formación de estos profesionales, desde un enfoque estratégico de prioridades del entorno, holístico (estudio en conjunto de manera integral de las partes de un todo), sistémico (todos los factores, actores y procesos interactúan y se complementan entre sí), antropocéntrico (todo lo que se hace es para y por las personas) con un enfoque inter, multi y transdisciplinario (con capacidad de interaccionismo social como gestores de procesos) con una visión ampliada del contexto actual y preparados para el futuro.

La universidad tiene el derecho y la obligación de transformarse, así como de modernizarse para ofrecer propuestas vigentes y pertinentes en el cometido histórico social depositado en ella por la sociedad, en lo correspondiente a la docencia, la investigación, la extensión de la cultura y los servicios y los procesos de vinculación (UNI, 2006, pág. 11).

El estudio de rendimiento académico es un tema que no solamente aborda lo clásico de las notas como un referente categórico sino que en éste interactúan factores sociales, personales, académicos e institucionales. Es necesario comprender que se está interactuando en un contexto lleno de cambios institucionales, de paradigmas y tecnológicos. Este es el contexto globalizado, competitivo y dinámico, donde el comportamiento social, las demandas y la sociedad en sí, así como la naturaleza cambian de manera vertiginosa.

El término rendimiento académico se refiere a uno de los procesos de mayor importancia para las instituciones universitarias, dado que representa un referente de la calidad de la educación y formación recibida por la comunidad estudiantil, incluyendo principalmente los procesos de enseñanza aprendizaje. Los factores que inciden para su evaluación e implementación son muchos, entre los más mencionados por diversos autores son los tecnológicos, socioeconómicos, interpersonales, institucionales, académicos y su interacción. A continuación, se establece un marco conceptual referencial de las diferentes perspectivas en que se abordado este tema.

Para Tonconi (2010) el rendimiento académico constituye un indicador del nivel de aprendizaje alcanzado por el estudiante y representa el nivel de eficacia en la consecución de los objetivos curriculares para las diversas asignaturas (Tonconi, 2010, pág. 1). 
Como refiere Reynoso (2011) son múltiples los factores considerados para determinar el desempeño académico de los estudiantes y se puede pensar que es aún más compleja la forma en cómo interactúan (Reynoso, 2011, pág. 33). Planteamiento que concuerda con el de Díaz (2010) quien afirma que no se puede hablar de calidad de la educación superior sin conocer a fondo indicadores asociados al rendimiento académico de los estudiantes universitarios, pues este análisis representa un monitoreo estratégico en cuanto al desempeño académico y por ende la utilización de los recursos que el Estado invierte" (Díaz, 2010, pág. 21). Las funciones de las universidades y los procesos universitarios deben estar orientados a la mejora continua de la calidad en la educación, tomando como referente las demandas del entorno. Debe de existir monitoreo y sistematización de las actividades orientadas a la mejora de todos los factores vinculados al rendimiento académico.

Según Montero (2007) el tema de los indicadores de rendimiento académico plantea varias interrogantes, entre ellas: ¿Cómo se puede construir una medida objetiva y fiable de todo lo que encierra el concepto de rendimiento académico?, ¿Existe realmente una medida cuantitativa y/o cualitativa del rendimiento de los y las estudiantes?, ¿Qué tipo de evaluación puede resultar más válida que las demás? (Montero, Jeannett, \& Astrid, 2007, pág. 217)

Para Hernández (2010) el proceso de formación profesional universitaria es complejo y exige el desarrollo de competencias académico profesionales y en esta misma dirección Herrera (1996) ya hacía referencia a que la formación de los profesionales debe descansar en la incorporación de mayores niveles de conocimiento, fomento del trabajo en equipo, capacidad de interacción simbólica, amplio conocimiento del proceso productivo, desarrollo de un pensamiento innovador y anticipatorio, y la construcción de mentalidades críticas y propositivas; asimismo, debe asegurar la constitución de equipos de trabajadores del conocimiento. Los estudiantes tienen la posibilidad de aprender todo lo que puedan con el fin de aplicar sus conocimientos en el sector productivo de la sociedad en los puestos de trabajo que adopten o en las empresas que establezcan (Alemán, Medrano, Nordren, Reyes, \& Scheinberg, 2010, p. 6).

Actualmente el uso de las tecnologías aplicadas a la educación es un factor determinante para los aprendizaje y de impacto en los resultados académicos reflejados en las notas obtenidas, en el caso de los estudiantes de ingeniería civil el desarrollo de prácticas paralelas a sus cursos fortalece sus habilidades para un mejor desempeño en el mundo laboral aumentado las probabilidades de empleabilidad en su formación. El establecimiento y aplicación de políticas, así como normativas y reglamentos de régimen académico que apoyan la labor docente, deben de estar acorde a un contexto caracterizado por los cambios en la gestión del conocimiento y tecnologías, donde los estudiantes se han caracterizado por el uso de Tecnologías de la Información y la Comunicación (TIC).

Martínez (2009) sugiere que la educación provee de información a los empleadores al señalizar y revelar los niveles de productividad del sujeto, siendo ésta su función principal. Por tanto, el nivel 
educativo es el medio de acceder al mercado laboral y de conseguir salarios adecuados. En su argumento también plantea que la enseñanza superior es considerada como un factor importante a la hora de determinar la inserción laboral, pero la procedencia de clase es la que posibilita y traza las trayectorias académicas y laborales de los sujetos, al determinar estrategias, actitudes, valores, discursos y expectativas, resultantes del proceso de socialización (Martínez, 2009, pág. 5).

Sobre el rendimiento académico la pregunta que en principio las instituciones de educación superior deberían responder es, ¿En el proceso de formación hubo factores relacionados al rendimiento académico que no fueron tomados en cuenta en la malla curricular? La respuesta a dicha pregunta debería conducir a la realización de acciones para generar más oportunidades para los futuros profesionales. Las tendencias recientes en el mundo del trabajo y la evolución de los sistemas educativos y de formación profesional indican que los jóvenes encuentran serias dificultades para lograr una inserción laboral y social plena (CIPPEC, 2009, pág. 7). El mercado laboral constituye un campo de gran interés desde el punto de vista económico, político y social. En efecto, los cambios que se generan en ésta área afectan a toda la sociedad, de ahí que los estudios que se realicen sobre el tema son siempre vigentes (Tobón \& Rodríguez, 2015, pág. 60).

Es necesario dejar de percibir el rendimiento académico desde un enfoque unidimensional que se refleja en una nota estricta cuantitativa en una escala evaluativa numérica, asociada generalmente a un índice de aprovechamiento del tiempo invertido en clase. El rendimiento académico es un resultado que va más allá de la interacción estudiante-docente como un proceso conexo que involucra elementos pedagógicos, didácticos, institucionales, así como psicoactivos reflejados idealmente en la culminación exitosa de los ciclos establecidos en los programas académicos.

Las calificaciones o notas obtenidas en test y pruebas han representado un criterio universal, institucional y social para evaluar el rendimiento académico de un estudiante y un indicador de su desempeño laboral, sin embargo según Alonso, Gil y Martínez (1996) estas valoraciones están sometidas a amplios márgenes de incertidumbre y afectan decisivamente aquello que se pretende medir. Los autores sugieren que la evaluación es la expresión de expectativas mayoritariamente subjetivas, que ejercen gran influencia sobre el comportamiento de los estudiantes y los profesores (Alonso, Gil, \& Martínez, 1996).

Guzmán (2012) indica que el rendimiento es lo necesario para que el alumno sea capaz de llevar a la práctica sus conocimientos, que pueda aplicar la información adquirida a la solución de problemas; en pocas palabras, es el resultado del trabajo escolar. Para Edel (2003) este es constructo susceptible de adoptar valores cuantitativos y cualitativos, a través de los cuales existe una aproximación a la evidencia y dimensión del perfil de habilidades, conocimientos, actitudes y valores desarrollados por el alumno en el proceso de enseñanza aprendizaje (Edel, 2003, Pg. 10). 
Para Muñoz (1977), citado por Guzmán (2012), no se consideraron en las definiciones anteriores factores que inciden en el éxito o fracaso escolar, como son las condiciones sociales, las actitudes, el esfuerzo del estudiante, etc. Todos estos aportes dieron como resultado una evolución del concepto del rendimiento académico (Guzmán, 2012, pág. 54). Garbanzo (2007) advierte al respecto que se debe diferenciar entre el rendimiento académico inmediato refiriéndose a las notas, y el mediato refiriéndose a los logros personales y profesionales (Garbanzo, 2007,pg. 46). Así, las calificaciones constituyen en sí mismas el criterio social y legal del rendimiento académico de un alumno o una alumna en el ámbito institucional (Montero, 2007, pg. 217).

Es ampliamente aceptado que el Rendimiento Académico refiere a una forma, escala o indicador empleado por casas de estudio para valorar la ubicación de un estudiante en distintos niveles académicos tanto de grado como de posgrado así como los aprendizajes alcanzados, pero como se ha indicado en los referentes teóricos encontrados, no es tan simple el abordaje de esos estudios ya que incluye una serie de factores llamados para unos internos (intrínsecos ) como externos (extrínsecos) y además una interrelación entre estos. Los factores cualitativos representan cierto nivel de dificultad en su determinación más que los cuantitativos, ya que abordan aspectos de índole social, académica, institucional, personal (aptitud y actitud desarrollada) y hasta del entorno en que se desarrollan las instituciones educativas.

Para Montes y Lerner (2011) el rendimiento académico representa la relación entre el proceso de aprendizaje, que involucra factores extrínsecos e intrínsecos al individuo, y el producto que se deriva de él, expresado tanto en valores predeterminados por un contexto sociocultural como en las decisiones y acciones del sujeto en relación con el conocimiento que se espera obtenga de dicho proceso (Montes \& Lerner, 2011, pág. 15).

Para Edel (2003) el rendimiento académico representa un constructo susceptible de adoptar valores cuantitativos y cualitativos, a través de los cuales existe una aproximación a la evidencia y dimensión del perfil de habilidades, conocimientos, actitudes y valores desarrollados por el alumno en el proceso de enseñanza aprendizaje (Edel, 2003, págs. 12-13).

Garbanzo (2007) refiere que el rendimiento académico del estudiantado universitario es un indicador que posibilita una aproximación a la realidad educativa y constituye un factor imprescindible en el abordaje del tema de la calidad de la educación superior (Garbanzo, 2007). En ese sentido, se hace necesario conocer a profundidad y de manera integral los resultados cuantitativos y cualitativos asociados al rendimiento académico, que permitan reflexionar sobre la práctica educativa y el impacto que se tiene en el entorno, además de validar el aporte de la universidad así como el cumplimento de su misión y visión, a fin de repensarse y reinventarse de acuerdo a las demandas de la sociedad. 
El rendimiento académico es complejo en su definición y forma de abordarlo, se modifica de acuerdo al objetivo del estudio y el enfoque puede ser amplio o limitado, tener aspectos netamente cuantitativos, cualitativos o de ambas perspectivas. El rendimiento académico como concepto y tema de estudio es dinámico y estático, pues responde al proceso de aprendizaje y se objetiva en un producto ligado a medidas y juicios de valor, según el modelo social vigente (Montes G. \& Lerner M., 2011, pág. 12)

Montes y Lerner (2011) hacen referencia a que los estudios realizados sobre el rendimiento académico permiten vislumbrar tres grupos o categorías de como ha venido entendiéndose esta temática. En el primer grupo se ha conceptualizado el rendimiento académico como un resultado expresado e interpretado cuantitativamente, en este se encuentran autores como Tonconi (2010) quien define el rendimiento académico como el nivel demostrado de conocimientos en un área o materia, evidenciado a través de indicadores cuantitativos, bajo el supuesto que es un "grupo social calificado" el que fija los rangos de aprobación, para áreas de conocimiento determinadas, para contenidos específicos o para asignaturas.

En el segundo grupo el rendimiento académico se ha definido como una forma expresada como juicio evaluativo - cuantificado o no sobre la formación académica, es decir, al proceso llevado a cabo por el estudiante considerando el proceso que pone en juego las aptitudes del estudiante ligadas a factores volitivos, afectivos y emocionales, además de la ejercitación para lograr objetivos o propósitos institucionales preestablecidos.

En el tercer grupo se considera que el rendimiento está expresado de manera combinada, asumiendo el rendimiento como proceso y resultado, evidenciado tanto en las calificaciones numéricas como en los juicios de valor sobre las capacidades y el 'saber hacer' del estudiante derivados del proceso $\mathrm{y}$, a su vez, teniendo en cuenta aspectos institucionales, sociales, familiares y personales de los estudiantes, los cuales afectan y son afectados en la dicotomía "éxito o fracaso académico". En este articulan horizontalmente las dos caras de rendimiento: proceso y resultado.

El resultado se expresa no sólo en notas sino también en acciones entendidas como lo que efectivamente el estudiante logra hacer con lo aprendido. De esta manera, el rendimiento académico es la expresión de capacidades y características psicológicas del estudiante que se actualizan a través de un proceso de aprendizaje (Montes G. \& Lerner M., 2011, págs. 12-13).

Guzmán (2012) indica que, con frecuencia, la medida del Rendimiento Académico de la enseñanza superior se determina por dos aspectos: el primero son las calificaciones que obtienen los alumnos a lo largo de los estudios hasta lograr la titulación correspondiente y el segundo hace referencia a los efectos que la formación recibida por los titulados tiene en la vida social; es decir, la utilidad que dichos estudios tienen en su proceso de incorporación al mundo laboral (Guzmán, 2012, pág. $83)$. 
Para que los estudios de rendimiento académico sean útiles, es importante identificar el tipo de influencia de los factores asociados al éxito o al fracaso del estudiantado evaluando las distintas categorías de variables personales, sociales e institucionales. La mayoría de estudios sobre rendimiento académico se basan en una aproximación metodológica de tipo predictivo, donde se utilizan modelos de regresión múltiple, pocas veces complementados con modelos explicativos que favorecen un análisis más integral de los factores asociados al rendimiento académico, por lo que es útil describir las características de los determinantes mencionados (Murillo, 2013, pág. 25). Como se refiere en el Modelo $\mathrm{I}+\mathrm{D}+\mathrm{i}$ de la UNAN-Managua, en esencia, los modelos para estudiar la realidad son una representación de muchos actores, factores y sus interacciones internas y externas, propias del contexto en que se vive, la visión prospectiva de los procesos de que se traten y el abordaje de los mismos (Pedroza, 2015, pág. 6).

Por su parte Garbanzo (2007) agrupa en tres grupos de determinantes que inciden en la calidad del rendimiento académico los cuales son mostrados en la Figura 1.

Figura 1. Interacción entre factores asociados al rendimiento académico

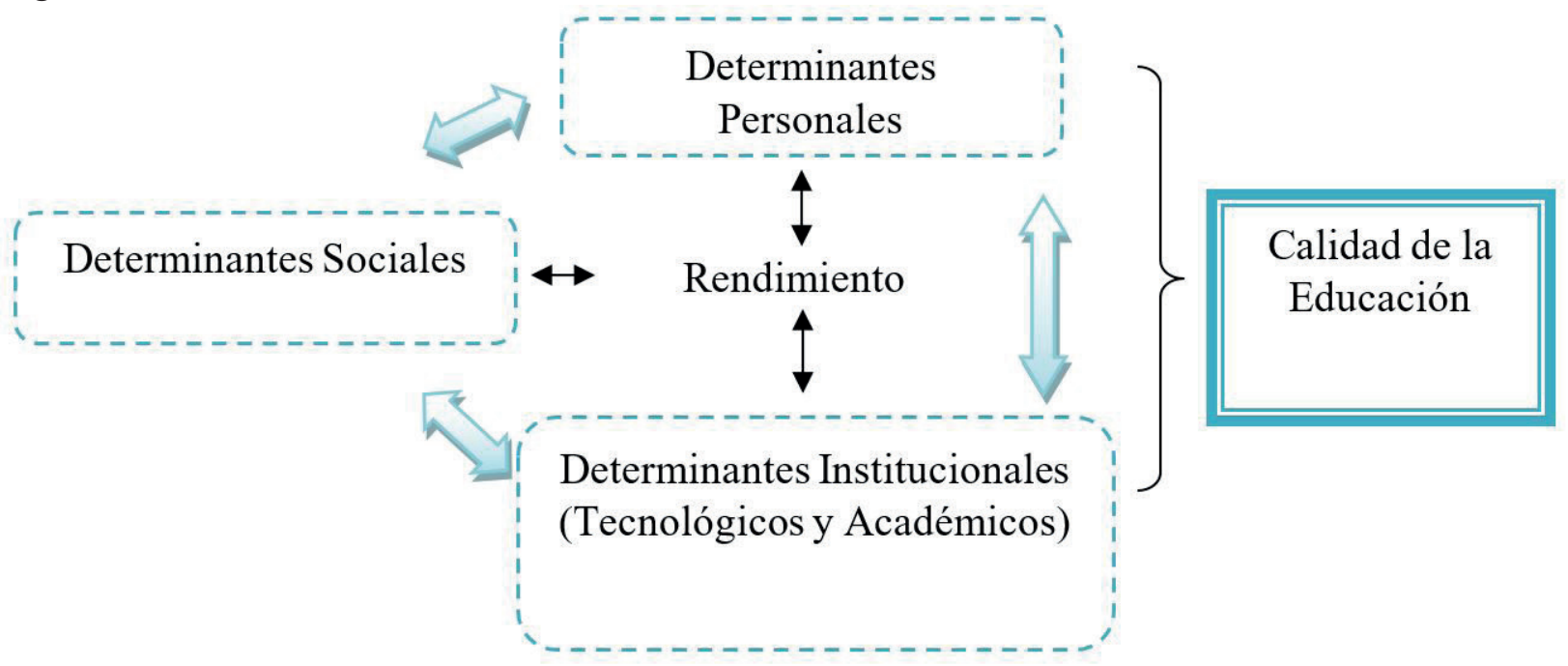

Fuente: (Garbanzo, 2007, pág. 60)

Los factores que inciden en el rendimiento académico de los estudiantes universitarios poseen una multidimensionalidad caracterizada en factores sociales, personales (Actitudes y aptitudes), institucionales, tecnológicas y académicas. En la Tabla 1 se muestran los factores determinantes en el rendimiento académico y las dimensiones de análisis que incluyen lo psicopedagógico, sociológico y fisiológico. 
Tabla 1: Factores determinantes en el rendimiento académico por dimensiones de análisis

\begin{tabular}{|c|c|}
\hline Dimensión & Factores \\
\hline Personales & 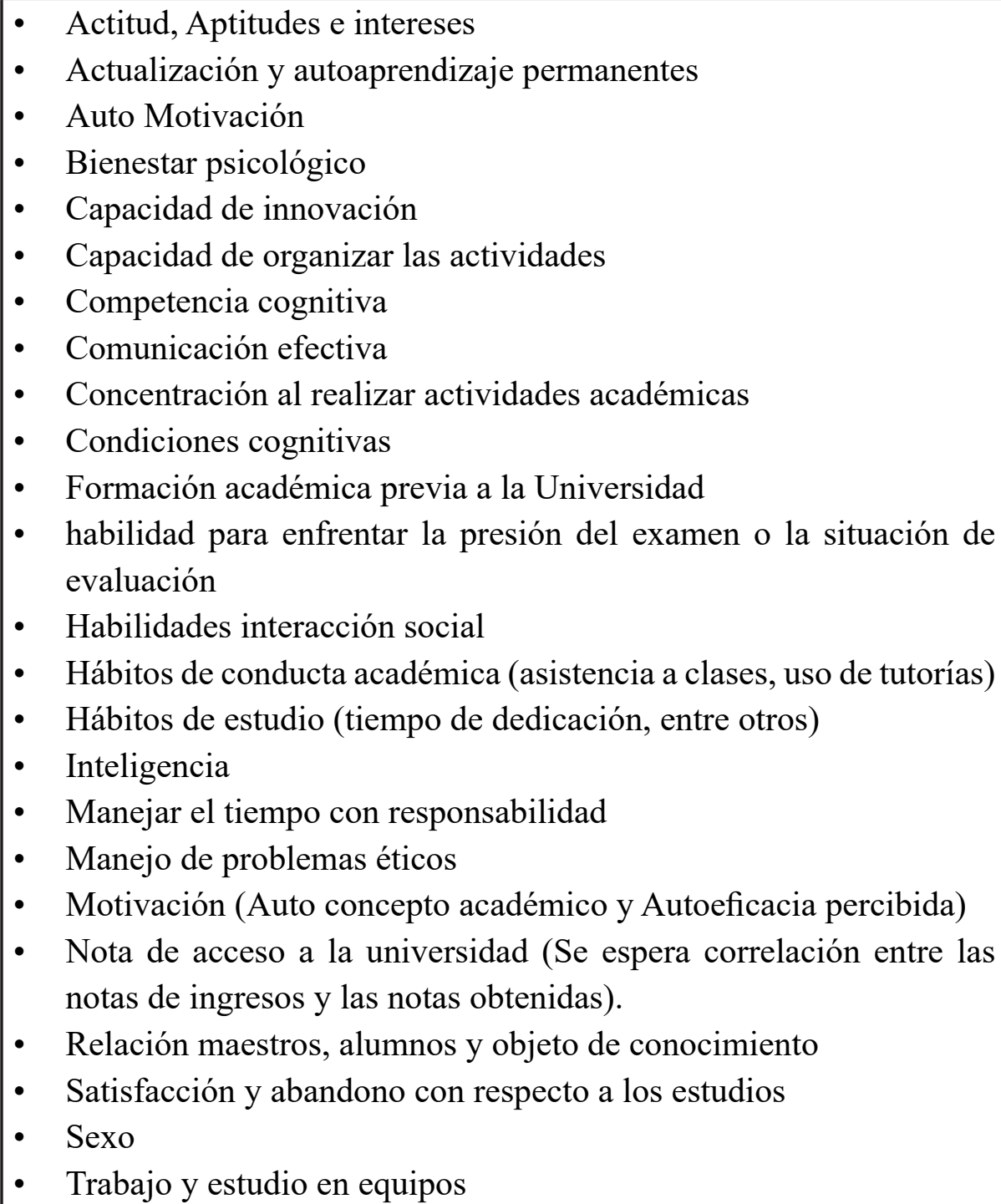 \\
\hline Académicos & $\begin{array}{l}\text { - } \quad \text { Experiencia o práctica complementaria a su perfil } \\
\text { - } \text { te línea curricular) } \\
\text { - Percepciones del estudiante (relación con los profesores, métodos } \\
\text { de enseñanza, percepción sobre su propio aprendizaje, y sobre las } \\
\text { ayudas y ambiente institucional, como informaciones auto-reporta- } \\
\text { das valoradas cualitativamente. }\end{array}$ \\
\hline
\end{tabular}




\begin{tabular}{|c|c|}
\hline Dimensión & Factores \\
\hline Sociales & $\begin{array}{l}\text { - Clima (Ambiente) y Entorno familiar } \\
\text { facer las necesidades (ingresos o fuentes de financiamiento para } \\
\text { vivienda, alimentación, vestuario, transporte, material de estudio, } \\
\text { gastos en actividades de esparcimiento y otros) } \\
\text { - Diferencias sociales } \\
\text { - Miembros de la familia en el hogar y estudiando } \\
\text { - Nivel educativo de los adultos responsables del estudiante } \\
\text { - Profesión y Ocupación de Padres } \\
\text { Variables demográficas (estrato y tipo de la vivienda) }\end{array}$ \\
\hline Institucionales & $\begin{array}{l}\text { - } \text { Ambiente estudiantil } \\
\text { - } \text { Cantidad docentes en actividades extracurriculares } \\
\text { - } \quad \text { Condiciones y recursos institucionales (la infraestructura física, he- } \\
\text { rramientas tecnológicas, laboratorios) } \\
\text { - Índice de eficiencia Terminal (relacionado a capacidad de } \\
\text { cumplimiento de su misión y visión) } \\
\text { - Elección de los estudios según interés del estudiante } \\
\text { - La experiencia y prácticas pedagógicas de los docentes } \\
\text { - } \quad \text { Pruebas específicas de ingreso a la carrera } \\
\text { - Vínculo con la experiencia laboral }\end{array}$ \\
\hline
\end{tabular}

Fuente: Elaborado a partir de Tobón (2015), Montes \& Lerner ( 2011), Ynzunza \& López (2011), Ocaña.(2011), Garbanzo(2007), Vargas (2004), CINDA (2004), Lerner y Gil (2002).

Es necesario que se cuente con una amplia gama de capacidades para el contexto laboral actual que cada vez exige la interdisciplinariedad con el trabajo de equipo que promueva la creatividad e innovación. En el caso particular de los ingenieros civiles, basados en el perfil de la carrera es necesario desarrollar la capacidad para aplicar criterios de urbanización en el diseño geométrico de vías, analizar el suelo de acuerdo a su tipo y capacidad de carga, calcular y diseñar los diferentes elementos estructurales de las obras, calcular y diseñar obras hidráulicas, regular la circulación de vehículos de carga o pasajeros de acuerdo al diseño del vehículo, planificar, verificar y ejecutar obras de acuerdo a planos y especificaciones técnicas, evaluar y formular proyectos de inversión. Núñez (2010) refiere que todos los conocimientos pueden ser útiles para el desarrollo; importan los conocimientos científicos y tecnológicos, las ciencias sociales, las humanidades. (Nuñez, 2010, pág. 169). Por otro lado, Ariza (2007) indica que la evaluación de competencias específicas sitúa a la motivación como el principal factor para la inserción laboral, seguido de la adaptabilidad a las condiciones cambiantes del entorno, la capacidad oral y escrita del candidato, su orientación hacia la calidad, la habilidad de organización y planificación y la capacidad de análisis y síntesis 
(Ariza, 2007, pág. 15). El planteamiento de estos autores coincide con la cantidad de factores determinantes de la calidad del rendimiento académico planteados en la Tabla 1, siendo los de mayor cantidad de indicadores los referidos a la dimensión personal.

Dentro del análisis de rendimiento académico deberá de ser retomado como insumo de análisis los reglamentos, normativas y políticas de la universidad que sirva como parámetro referencial de medición del concepto y aplicación cuantitativa.

La tecnología ha cambiado la valoración de las cualidades de los trabajadores en las empresas, aquí y para la referencia de este artículo se acepta que el término tecnología, basado en Sábato (2011) y CONICYT (2008), se refiere al conjunto de conocimientos desarrollados y estructurados que permitan la creación de bienes o servicios tangibles e intangibles adaptados a las necesidades sociales y humanas que permitan el aprovechamiento con eficiencia y eficacia los recursos humanos así como materiales.

Como parte de las correlaciones existentes entre los factores de rendimiento académico desde su multidimensionalidad, con lo referido a la empleabilidad de los egresados y graduados de las carreras de ingeniería, que permita establecer planes de mejora prospectivos se hace necesario definir lo que implica la empleabilidad desde el enfoque de otros investigadores.

El término "empleabilidad" se refiere a las competencias y cualificaciones transferibles que refuerzan la capacidad de las personas para aprovechar las oportunidades de educación y de formación que se les presenten con miras a encontrar y conservar un trabajo, progresar en la empresa o al cambiar de empleo y adaptarse a la evolución de la tecnología y de las condiciones del mercado de trabajo (OIT, 2005, pág. 3). Para Baptista y Dos Santos (2016) en referencia a Sarsur (1999) la empleabilidad se entiende como: Una acción individual, que puede ser estimulada o no por las organizaciones, que hace que los empleados de todos los niveles busquen estar mejor preparados para enfrentar el mercado de trabajo y sus cambios, que presupone una postura proactiva, en el sentido de capacitarse y cualificarse permanentemente, en términos de habilidades y capacidades técnicas, humanas, conceptuales y de relaciones sociales (Baptista \& Dos Santos, 2016, p. 37). Para EAE, Escuela de Negocios de España, en referencia a Factor Humano, en la actualidad los aspectos clave que mejoran la empleabilidad en un mundo laboral cada vez más tecnificado y en constante evolución son la adaptabilidad, flexibilidad, creatividad, habilidad social, competencia digital, iniciativa y pasión, liderazgo (EAE, 2016).

Pérez (2014) refiere a que la empleabilidad podrá ser visualizada como la capacidad desde el individuo para la obtención y mantención de un empleo con sus habilidades, conocimientos y aptitudes así como actitudes, desempeñándose en distintos roles. Además, señala que Yorke (2004) refiere a la empleabilidad de los graduados universitarios como un conjunto de logros, habilidades, conocimientos y atributos personales- que aumentan la probabilidad de los graduados de encontrar 
un empleo y de tener éxito en sus puestos, lo cual beneficia a ellos mismos, a la fuerza de trabajo, a la comunidad y a la economía (Pérez, 2014, pág. 187).

Para Baptista y Dos Santos (2016) la empleabilidad se puede entender como las acciones emprendidas por las personas para desarrollar habilidades y buscar conocimientos favorables, con el objetivo de lograr una posición en el mercado de trabajo, sea en el formal sea en el informal (Baptista \& Dos Santos, 2016, p. 1).

La empleabilidad es entonces la ubicación de estudiantes en puestos de trabajo que respondan a su perfil profesional que apliquen los conocimientos desarrollados a lo largo del desarrollo de su vida académica en la Institución educativa de educación superior. Para un estudio de la empleabilidad se ha de considerar y prestar atención al hecho de que, quizás, muchos de los miembros de una comunidad estudiantil no trabajan bajo el perfil de su formación, sino bajo otros contextos como propio negocio o perfiles de otras carreras siendo un criterio de exclusión para el análisis.

Para Muñoz (2006) es necesario lograr una suficiente correspondencia entre las características de esa formación y los requerimientos de las ocupaciones, conocimientos, competencias y actitudes necesarios para trabajar exitosamente en su profesión, así como un razonable equilibrio entre las cantidades de jóvenes que egresan de las IES y la capacidad del sistema económico para absorberlos en condiciones aceptables (Muñoz I., 2006, p. 2).

La Universidad moderna requiere de una permanente revisión de las necesidades de su entorno, la creación de redes académicas y sociales sólidas y la creación de asociaciones intermediarias de interfaz (Pineda, Morales, \& Ortiz, 2011, pág. 62).

El desarrollo de la empleabilidad es de mucha importancia, pues pretende mantener el profesional apto para incorporarse al mercado de trabajo. Sin embargo, para desarrollarse el individuo necesita estar en constante búsqueda de conocimiento y experiencias que le servirán de bagajes para competir frente a las exigencias del mercado. Las tecnologías de Información y Comunicación como parte del diseño Curricular implican que estarán al servicio del proceso enseñanza-aprendizaje, donde hoy por hoy las redes sociales son las más usadas por los jóvenes y se puede lograr que estas ayuden a potenciar la calidad educativa (CNU, 2016).

De hecho, en el ámbito de las Universidades se contemplan algunas acciones concretas para mejorar la empleabilidad de los estudiantes como son la participación de profesionales en contextos educativos, el fomento de las prácticas y la orientación profesional, articulados en torno a diseñar la carrera con su correspondiente perfil profesional y la orientación hacia el empleo (Pérez, 2014, págs. 88-89). 
Lo anterior concuerda con los lineamientos y estrategias del Gobierno de Nicaragua en pro del desarrollo social con perspectivas de desarrollo y equidad. Tal es así que con el fin de seguir acordando acciones estratégicas que permitan mejorar la Educación Superior orientada a elevar la calidad y pertinencia de la Educación Superior Pública fortaleciendo la institucionalidad y la articulación de la Educación Superior, con los distintos sectores de la sociedad, procurando mayor eficiencia, eficacia y efectividad en su quehacer; crear una cultura de rendición social de cuentas, incluyendo las contribuciones de las universidades a resolver la problemática nacional, sobre todo aquellas que reproducen los círculos viciosos de exclusión y pobreza de las mayorías (Gobierno de Reconciliación y Unidad Nacional, 2012, pág. 81).

Finalmente, para los lectores y críticos de esta temática vale la pena hacer referencia a los resultados de aprendizaje esperados al término de la carrera, establecido por marco de cualificaciones para la educación superior centroamericana, que sirven de referente para la evaluación y análisis de los factores que inciden en el rendimiento académico y que genera punto de inflexiones para la planificación estratégica de las Instituciones educativas superiores los cuales se muestran en Tabla 2.

Tabla 2. Resultados de aprendizajes esperado para los estudiantes de nivel de licenciatura

\begin{tabular}{|l|l|}
\hline \multicolumn{1}{|c|}{ Descriptor } & \multicolumn{1}{c|}{ Resultados de aprendizaje esperados } \\
\hline $\begin{array}{l}\text { Saberes } \\
\text { disciplinarios y } \\
\text { profesionales }\end{array}$ & $\begin{array}{l}\text { Comprende el cuerpo conceptual, metodológico y procedimental } \\
\text { actualizado que le brinda identidad y fundamento a su profesión en el } \\
\text { contexto nacional, regional e internacional. } \\
\text { Posee conocimientos de áreas complementarias como gestión del riesgo, } \\
\text { medio ambiente, cultura y derechos fundamentales, que le brindan una } \\
\text { visión amplia de su profesión, de las relaciones de esta con otros saberes } \\
\text { y de su entorno. }\end{array}$ \\
\hline $\begin{array}{l}\text { Aplicación de } \\
\text { conocimientos, } \\
\text { información y } \\
\text { resolución de } \\
\text { problemas e } \\
\text { innovación }\end{array}$ & $\begin{array}{l}\text { Utiliza los conocimientos de su disciplina en la elaboración, } \\
\text { fundamentación y defensa de argumentos para prevenir y resolver } \\
\text { problemas en su campo profesional, identificando y aplicando } \\
\text { innovaciones. }\end{array}$ \\
$\begin{array}{l}\text { Utiliza de forma adecuada las técnicas, protocolos y tecnologías } \\
\text { pertinentes a su disciplina o campo profesional. } \\
\text { Demuestra capacidad y actitud investigativa que le permiten la } \\
\text { identificación, descripción, análisis crítico y explicación de problemas. } \\
\text { Identifica, aborda y desarrolla opciones de solución a problemas en su } \\
\text { campo profesional o disciplina. } \\
\text { Identifica oportunidades y riesgos para la innovación y adaptación de } \\
\text { conocimientos y tecnologías para resolver problemas. } \\
\text { Identifica, analiza y gestiona el riesgo en el ejercicio de su profesión. }\end{array}$ \\
\hline
\end{tabular}




\begin{tabular}{|c|c|}
\hline Descriptor & Resultados de aprendizaje esperados \\
\hline $\begin{array}{l}\text { Autonomía con } \\
\text { responsabilidad } \\
\text { personal, } \\
\text { profesional y } \\
\text { social }\end{array}$ & $\begin{array}{l}\text { - Demuestra capacidad para ejercer su profesión en forma responsable } \\
\text { dentro de los marcos normativos y éticos de su campo profesional, y } \\
\text { reflexiona sobre la incidencia de sus decisiones en los aspectos humanos, } \\
\text { sociales y ambientales. } \\
\text { Muestra iniciativa y capacidad para emprender nuevos proyectos } \\
\text { profesionales para la generación de negocios, o para el beneficio social } \\
\text { con visión de sostenibilidad económica, social y ambiental. } \\
\text { - Asume compromiso con su aprendizaje permanente y posee la capacidad } \\
\text { para identificar sus necesidades de formación en su campo de estudio } \\
\text { y entorno profesional, así como las formas de abordar su actualización. } \\
\text { Asume compromiso y una actitud participativa y propositiva en acciones } \\
\text { y decisiones que protegen y mejoran la vida personal y de su comunidad } \\
\text { y de su país, incidiendo en la reducción de su vulnerabilidad y la de } \\
\text { su entorno. Asimismo, promueve estos principios en el marco de su } \\
\text { ejercicio profesional. }\end{array}$ \\
\hline Comunicación & $\begin{array}{l}\text { - Habilidad para estructurar argumentos y mensajes adecuados a diversos } \\
\text { públicos y comunicarlos con claridad, rigurosidad y precisión, haciendo } \\
\text { un su apropiado del lenguaje: escrito, verbal, numérico, gráfico y } \\
\text { multimedia, en distintas lenguas. }\end{array}$ \\
\hline $\begin{array}{l}\text { Interacción } \\
\text { profesional, } \\
\text { cultural y social }\end{array}$ & $\begin{array}{l}\text { - Trabaja en espacios profesionales, comunitarios e interculturales de } \\
\text { forma colaborativa, para la solución de problemas y el logro de metas } \\
\text { conjuntas, con ética, respeto y solidaridad. }\end{array}$ \\
\hline
\end{tabular}

Fuente: (CSUCA, 2013, p. 18)

\section{CONCLUSIONES}

Acorde a la sociedad en que se vive y desarrollo del contexto de cambios de tecnología y actualización de conocimiento es relevante tomar el cuanto los factores personales, sociales, tecnológicos e institucionales relacionados al rendimiento académico como productos del proceso de enseñanza aprendizaje. De múltiples contextualizaciones el rendimiento académico se ha considerado principalmente como una escala numérica de las posibles capacidades del estudiante, sin embargo la realidad es no sólo es una nota cuantitativa la que refleja los aprendizajes en los formados en ingeniería, siendo necesario una caracterización y evaluación de los factores objetivos relacionados con los niveles de empleabilidad que permita establecer lineamientos y políticas estratégicas que contribuyan a corto, mediano y largo plazo en la mejora de los procesos inserción laboral, pudiendo hacer una evaluación de los aportes de las instituciones educativas a través de sus formados. Es necesario identificar para un contexto regional cuales han sido los factores más determinantes que inciden en la calidad del rendimiento académico de los estudiantes de ingeniería. 
Es una demanda de la sociedad para las instituciones educativas el dotar de competencias integrales y habilidades, así como actitudes propias de programa académico de ingeniería que permitan aumentar las posibilidades de ser empleado, contribuyendo al aumento de la productividad, optimización de recursos que permita desde lo local al desarrollo de la región. En la evolución de las ingenierías no ha habido cambios transcendentales en el sistema de evaluación del rendimiento académico desde el enfoque cualitativo. Se considera que no se incluyen factores de orden cualitativo en normativas y reglamentos vigentes que se reflejen en las habilidades, competencias, actitudes, aptitudes y experiencias vividas por lo estudiantes que se reflejen en el desempeño laboral.

\section{REFERENCIAS BIBLIOGRÁFICAS}

Alemán, F., Medrano, H., Nordren, A., Reyes, A., \& Scheinberg, S. (2010). Innovaciones del CNU. 2010: Universidad Nacional Agraria, UNA.

Alonso, M., Gil, D., \& Martínez, J. (1996). Evaluar no es calificar. La evaluación y la calificación en una enseñanza constructiva de las ciencias. Revista Investigación en la Escuela, 30, 15-26.

Ariza Montes, J. A. (2007). Las competencias: un factor clave en la empleabilidad de los titulados Universitarios. Revista de Fomento Social , 15.

Baptista, A., \& Dos Santos, R. (2016). Empleabilidad de los estudiantes de grado tras la conclusón del curso. Um estudio em las "faculdade de tecnologia de são paulo" - "ZONA LESTE" Y "GUARULHOS". SADSJ - South American Development Society Journal, 14.

CINDA. (2004). Competencias Estudiantes Universitarios. Satiago, Chile: Centro interuniversitario de Desarrollo-cINDA.

CIPPEC. (2009). Los jóvenes y la formación para el trabajo en América Latina. Buenos Aires, Argentina: Centro de Implementación de Políticas Públicas para la Equidad y El crecimiento. CIPPEC.

CNU. (9 de Febrero de 2016). Martínez Toribio, Juan José . Las TICs en las universidades del CNU. Managua. Obtenido de http://www.cnu.edu.ni/las-tics-en-las-universidades-del-cnu/

CSUCA. (2013). Marco de cualificaciones para la educación superior centroamericana. Resultados de aprendizaje para licenciatura, maestría y doctorado. Una aproximación desde la visión académica. Guatemala: Consejo Superior Universitario Centroamericano (CSUCA).

Díaz de Iparraguirre, A. (2009). La gestión compartida universidad-empresa en la formación del capital humano. Su relación con la promoción de la competitividad y el desarrollo sostenible. Caracas, Venezuela: Universidad Nacional Experimental Simón Rodríguez .

Díaz, A. V. (2010). Incidencia de los Estudiantes Monitores, en el rendimiento académico de los educandos que cursan la asignatura de Matemática IV, de la Carrera de Ingeniería Civil, en la UNI-Norte, II Semestre 2009. . Managua: Universidad Nacional de Ingeniería.

CSUCA (2013). Consejo Superior Universitario Centroamericano. Marco de cualificaciones para la educación superior centroamericana. Resultados de aprendizaje para licenciatura, maestría y doctorado. Alfa Puentes. Guatemala.

EAE, B. S. (12 de Agosto de 2016). EAE Business School. Obtenido de http://www.eaeprogramas. es/blog/los-siete-factores-clave-de-la-empleabilidad-en-un-mundo-laboral-tecnologico 
Edel, R.. (2003). El rendimiento académico: concepto, investigación y desarrollo. REICE - Revista Electrónica Iberoamericana sobre Calidad, Eficacia y Cambio en Educación , 15.

Garbanzo, G. (2007). Factores asociados al rendimiento académico en estudiantes universitarios, una reflexión desde la calidad de la educación superior pública. Revista Educación 31, ISSN: 0379-7082, 43-63,.

Gobierno de Reconciliación y Unidad Nacional. (2012). Gobierno de Reconciliación y Unidad Nacional - Plan Nacional de Desarrollo Humano 2012-2016. Managua: Consejo de Comunicación y Ciudadania.

Guzmán, M. (2012). Modelos predictivos y explicativos del rendimiento académico universitario: caso de una institución privada en México. Madrid: Universidad Complutense de Madrid.

Hernández M., E. M. (2010). Aprendizajes, competencias y rendimiento académico en latitulación de estudios socioculturales de la universidad de cienfuegos. Granada: Universidad de Granada.

Irigoin, M., \& Fernando, V. (2002). Competencia Laboral, Manual de conceptos, métodos y aplicaciones zen el Sector Salud. Montevideo: OPS/OMS. Obtenido de http://oitcinterfor.org/ sites/default/files/file_publicacion/man_ops.pdf

Izar L., J. M., Ynzunza, C. B., \& López, H. (2011). Factores que afectan el desempeño académico de los estudiantes de nivel superior en Rioverde, San Luis Potosí, México. Revista de Investigación Educativa 12, 8.

Martínez (2009). El proceso de inserción laboral: Implicaciones educativas para la mejora de la empleabilidad. Universidad de Granada, 17.

Montes G., I. C., \& Lerner M., J. (2011). Rendimiento académico de los estudiantes de pregrado de la Universidad EAFIT. Perspectiva Cuantitativa. Colombia: Universidad EAFIT. Obtenido de http://www.eafit.edu.co/institucional/calidad-eafit/investigacion/Documents/ Rendimiento\%20Ac\%C3\%A1demico-Perrspectiva\%20cuantitativa.pdf

Muñoz, C. (2006). Determinantes de la empleabilidad de los jóvenes universitarios y alternativas para promoverla. Scielo, 9.

Murillo, E. (2013). Factores que inciden en el Rendimiento Académico en el área de Matemáticas de los estudiantes de noveno grado en los centros de Educación Básica de la Ciudad de Tela, Atlándita. Universidad Pedagógica Nacional, San Pedro Sula, Honduras.

Montero, E., Jeannett, V., \& Astrid, V. (2007). Factores Institucionales, Pedagógicos, Psicosociales y Sociodemográficos asociados al rendimiento académicos en la Universidad de Costa Rica: Un Análisis Multinivel . Revista ELectrónica de Investigación y evaluación educativa - Relieve, 20.

Nuñez Jover, J. (2010). Conocimiento académico y sociedad Ensayos sobre política universitaria de investigación y posgrado. La Habana: UH.

Ocaña, Y. (2011). Variables académicas que influyen en el rendimiento académico de los estudiantes universitarios. Investigación Educativa , 165-179.

OIT (2005). Ministerio de trabajo, empleo y Seguridad Social, Dirección Nacional de Orientación y Formación Profesional la Formación Profesional. Argentina: Programa de Calidad del Empleo y Seguridad Social. Resolución 195 - OIT. Obtenido de http:/www.trabajo.gob.ar/downloads/ calidad/internacional_res195-oit.pdf 
Pedroza, (2015). Curso de Metodología de Investigación Científica. MEDINV I Cohorte. . Managua: UNAN- Managua.

Pedroza, (2016). Curso de Metodología de la Investigación. DOGCINV I Cohorte. Estelí, UNAN - Managua.

Pedroza, H (2015). El Modelo de Gestión de la Investigación, Modelo I+D+i de la UNANManagua. Managua: UNAN.

Pérez, M. (2014). Las prácticas externas y el Espacio Europeo de Educación Superior: análisis del desarrollo de competencias genéricas. Madrid: Universidad Europea Madrid.

Pineda, K., Morales, E., \& Ortiz, M. (2011). Modelos y mecanismos de interacción universidadempresa-Estado: retos para las universidades colombianas. Proyecto Institucional Integración Universidad,Empresa y Estado. Granada: Universidad Militar Nueva Granada.

Reynoso, E. (2011). Factores que determinan el rendimiento escolar en el nivel secundario en el estado de nuevo leon . Mexico.

Sabato, J. (2011). El pensamiento latinoamericano en la problemática ciencia-tecnología desarrollodependencia. Buenos Aires: Ediciones Biblioteca Nacional.

Tobón \& Rodríguez, F. (2015). Factores que determinan la probabilidad de participación laboral en el area metropolitana de Medellín. Medellín, Colombia: Universidad EAFIT.

Tonconi, J. (2010). Factores que influyen en el rendimiento académico y la deserción de los estudiantes de la facultad de ingeniería económica de la una-puno, periodo 2009. Cuadernos de Educación y Desarrollo - EUMED, 23.

UNI, Universidad Nacional de Ingeniería (2008), Modelo Educativo Institucional, Managua. Nicaragua

Vargas Z, F. (2004). Competencias Clave y aprendizaje permanente - Tres miradas a su desarrollo. Montevideo: Oficina Internacional del Trabajo - CINTERFOR. 